\title{
COSMOLOGY FROM XMM HIGH-Z AGN CLUSTERING
}

\author{
Manolis Plionis ${ }^{1,2} \&$ Spyros Basilakos ${ }^{3}$ \\ 1 Institute of Astronomy $\&$ Astrophysics, National Observatory of Athens, Greece \\ 2 INAOE, Puebla, Mexico \\ 3 Academy of Athens, Athens, Greece
}

\begin{abstract}
We review the high-redshift X-ray selected AGN clustering, based on the $\mathrm{XMM} / 2 \mathrm{dF}$ survey, and compare it with other recent XMM-based results. Using the recent Hasinger et al. (2005) and La Franca et al. (2005) luminosity functions we find that the spatial clustering lengths, derived using Limber's inversion equation, are $\sim 17$ and $20 h^{-1} \mathrm{Mpc}$ for the soft and hard band sources while their median redshifts are $\bar{z} \sim 1.2$ and 0.8 , respectively. The corresponding bias factors at $z=\bar{z}$ are $\sim 5.3 \pm 0.9$ and $\sim 5.1 \pm 1.1$, respectively. Within the framework of a flat cosmological model we find that our results support a model with $\Omega_{m} \simeq 0.26, \sigma_{8} \simeq 0.75, h \simeq 0.72, \mathrm{w} \simeq-0.9$ (in excellent agreement with the 3 year WMAP results). We also find the present day bias of X-ray AGNs to be $b_{\circ} \sim 2$.
\end{abstract}

\section{Introduction}

Active Galactic Nuclei (AGN) can be detected out to high redshifts and thus their clustering properties can provide information on the large scale structure, the underlying matter distribution and the evolution with redshift of the AGN phenomenon. Optically selected AGN surveys miss large numbers of dusty systems and therefore provide a biased census of the AGN phenomenon. X-ray surveys, are least affected by dust providing an efficient tool for compiling uncensored AGN samples over a wide redshift range.

Early studies of the X-ray AGN clustering, using Einstein and ROSAT data, produced contradictory results $\left(\right.$ eg. $\left.;^{9} ;{ }^{43} ;{ }^{11} ;{ }^{131}\right)$. Recently, there has been an effort to address this confusing issue and determine the clustering properties of both soft and hard X-ray selected AGNs, based on the new XMM and Chandra missions (eg. $;^{46} ; 47 ; 7,{ }^{8},{ }^{21} ;{ }^{36} ;{ }^{1730}$ ). Most of these studies find a relatively large correlation length for the high-z X-ray AGNs $\left(\right.$ eg.,, $\left.7^{7} ;{ }^{836}\right)$. However, the recent XMM-COSMOS survey results ${ }^{30}$ provided significantly smaller clustering lengths, opening again the contradiction realm. 


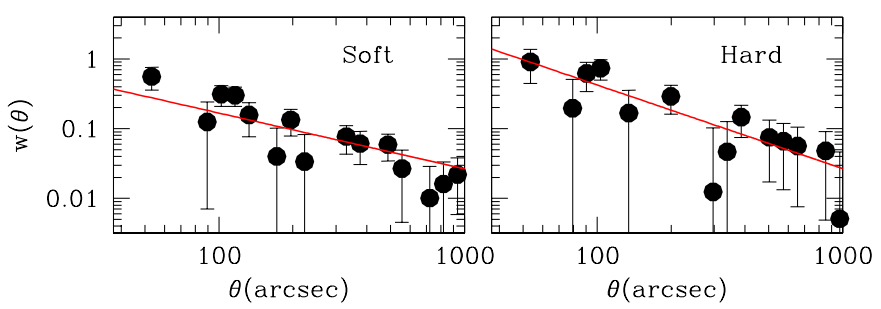

Fig. 1. The XMM/2dF survey angular correlation function for the 2 bands considered.

\section{The $X M M / 2 d f$ survey angular and spatial clustering}

The XMM-Newton/2dF survey is a shallow (2-10 ksec per pointing) survey comprising of 18 XMM-Newton pointings equally split between a Northern and Southern Galactic region.Due to elevated particle background we analysed a total of 13 pointings. A full description of the data reduction, source detection and flux estimation are presented in Georgakakis et al., ${ }^{1918}$

We derive the source $\log N-\log S$ after constructing sensitivity maps in order to estimate the area of the survey accessible to point sources above a given flux limit $\left(\right.$ see $^{78}$ ). In table 1 we also provide the effective flux-limit of the different surveys, estimated using the corresponding area curves, by: $S_{\mathrm{lim}}^{\mathrm{eff}} \simeq \sum \Omega_{i} S_{\mathrm{lim}, \mathrm{i}} / \sum \Omega_{i}$, where $\Omega_{i}$ is the survey solid angle of which the flux limit is $S_{\text {lim,i. }}$.

We calculate the angular correlation function using the estimator: $\mathrm{w}(\theta)=f\left(N_{D D} / N_{D R}\right)-1$, of which the uncertainty is: $\sigma_{w}=$ $\sqrt{(1+\mathrm{w}(\theta)) / N_{D R}}$, where $N_{D D}$ and $N_{D R}$ are the number of data-data and data-random pairs, respectively, in the interval $[\theta-\Delta \theta, \theta+\Delta \theta]$. The normalization factor is $f=2 N_{R} /\left(N_{D}-1\right)$, with $N_{D}$ and $N_{R}$ the total number of data and random points, respectively. For each XMM pointing we produce 100 Monte Carlo random catalogues having the same number of points as the real data accounting also for the sensitivity variations across the surveyed area (for details see, ${ }^{78}$ ). We use a standard $\chi^{2}$ minimization procedure to fit the measured correlation function assuming a power-law form: $\mathrm{w}(\theta)=\left(\theta_{\circ} / \theta\right)^{\gamma-1}$ and fixing $\gamma$ to 1.8. The fitting is performed for angular separations in the range $40^{\prime \prime}-1000^{\prime \prime}$ The resulting raw values of $\theta_{\circ}$ are corrected for the integral constraint and the amplification bias (for details see ${ }^{43}$ and $^{8}$ ), although such corrections are quite small. The final results are presented in Table 1. Now, the spatial correlation function can be modeled as (eg. $\left.{ }^{14}\right): \xi(r, z)=\left(r / r_{\circ}\right)^{-\gamma} \times(1+z)^{-(3+\epsilon)}$, where $\epsilon$ parametrizes the clustering evolution model. For $\epsilon=\gamma-3$ (ie., $\epsilon=-1.2$ for $\gamma=1.8$ ), 


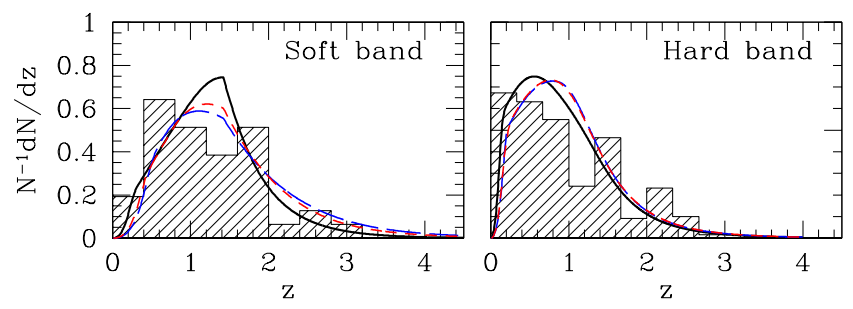

Fig. 2. Left Panel: The expected $z$-distributions using the Hasinger et $\mathrm{al}^{23}$ soft-band luminosity function for the XMM/2dF survey (thick line), the XMM-COSMOS survey (blue long-dashed line) and the XMM-ELAIS-S1 survey (red short-dashed line). Right Panel: The corresponding redshift distributions using the LDDE hard-band luminosity function of La Franca et al. ${ }^{28}$ The histogram in the left panel corresponds to the distribution of the X-ray sources in the ROSAT Lochman Deep Field, ${ }^{39}$ while that of the right panel on limited spectroscopic and photo-z data of our XMM/2dF survey.

the clustering is constant in comoving coordinates, a model which appears to be appropriate for active galaxies $\left(\right.$ eg. ${ }^{26}$ ).

In order to invert the angular correlation function to three dimensions we utilize Limber's integral equation $\left(\mathrm{eg} .{ }^{34}\right)$. For a spatially flat Universe, Limber equation can be written as:

$$
\mathrm{w}(\theta)=2 \frac{H_{\circ}}{c} \int_{0}^{\infty}\left(\frac{1}{N} \frac{\mathrm{d} N}{\mathrm{~d} z}\right)^{2} E(z) \mathrm{d} z \int_{0}^{\infty} \xi(r, z) \mathrm{d} u
$$

where the number of objects in a survey of a solid angle $\Omega_{s}$ and within the shell $(z, z+\mathrm{d} z)$, is given by:

$$
\frac{\mathrm{d} N}{\mathrm{~d} z}=\Omega_{s} x^{2} \phi(x)\left(\frac{c}{H_{\circ}}\right) E^{-1}(z)
$$

with $\phi(x)$ the selection function and $x$ the proper distance related to the redshift through $\left(\mathrm{see}^{34}\right)$ :

$$
x(z)=\frac{c}{H_{\circ}} \int_{0}^{z} \frac{\mathrm{d} u}{E(u)} \quad \text { with } \quad E(z)=\left[\Omega_{\mathrm{m}}(1+z)^{3}+\Omega_{\Lambda}\right]^{1 / 2},
$$

Since we do not have complete redshift information for our sources we estimate $\mathrm{d} N / \mathrm{d} z$ using the X-ray source luminosity function and folding in the area curve, via the relation: $\phi(x)=\int_{L_{\min }(z)}^{\infty} \Phi\left(L_{x}, z\right) \mathrm{d} L$, where $\Phi\left(L_{x}, z\right)$ is the luminosity dependent density evolution luminosity (LDDE) function. In the present analysis we use that of Hasinger et al. ${ }^{23}$ for the soft-band and that of La Franca et al. ${ }^{28}$ for the hard-band.

In Fig. 2 we present the expected redshift distributions of the soft and hard X-ray sources for all three recent XMM based surveys together with 
Table 1. Correlation function analysis results with their $1 \sigma$ uncertainties for the different surveys and X-ray bands. The correlation lengths assume a slope $\gamma=1.8$.

\begin{tabular}{ccccccc}
\hline Band $(\mathrm{keV})$ & Survey & $\#$ & $S_{\lim }^{\text {eff }}$ & $\theta_{\circ}\left({ }^{\prime \prime}\right)$ & $r_{\circ}\left(h^{-1} \mathrm{Mpc}\right)$ & $\bar{z}$ \\
\hline $0.5-2$ & XMM/2dF & 432 & $1.3 \times 10^{-14}$ & $10.4 \pm 3$ & $17 \pm 2.2$ & 1.25 \\
$0.5-2$ & XMM-COSMOS & 1037 & $1.9 \times 10^{-15}$ & $1.9 \pm 0.3$ & $9.3 \pm 0.7$ & 1.33 \\
$0.5-2$ & XMM-ELAIS & 395 & $4.5 \times 10^{-15}$ & $5.2 \pm 3.8$ & $13.8 \pm 4.5$ & 1.32 \\
$2-8$ & XMM/2dF & 177 & $4.6 \times 10^{-14}$ & $22.2 \pm 9$ & $20 \pm 3.7$ & 0.80 \\
$4.5-10$ & XMM-COSMOS & 151 & $1.6 \times 10^{-14}$ & $6 \pm 2$ & $12 \pm 1.8$ & 0.89 \\
$2-10$ & XMM-ELAIS & 205 & $1.8 \times 10^{-14}$ & $12.8 \pm 7.8$ & $17 \pm 4.6$ & 0.88 \\
\hline
\end{tabular}

the histogram of some limited spectroscopic and photo-z data (see caption for details). It is evident that all three surveys trace similar depths although the XMM-COSMO and XMM-ELAIS surveys have a slightly larger contribution from $z \gtrsim 2$ in the soft-band. The predicted median redshift of the three XMM surveys for the soft and hard sources are shown in table 1.

Then the inversion of eq.(2), using the LDDE luminosity function, $\epsilon=$ -1.2 , the concordance cosmological model, and integrating out to $z=4.5$, provides a correlation length of $r_{\circ} \simeq 17 \pm 2.2 h^{-1} \mathrm{Mpc}$ and $\simeq 20 \pm 3.7 h^{-1}$ $\mathrm{Mpc}$, for the soft and hard bands, respectively (the slight differences with respect to the Basilakos et al. ${ }^{78}$ results are due to the different luminosity functions used and a better treatment of the errors). These results are in good agreement with the XMM-ELAIS results ${ }^{36}$ but significantly larger than the XMM-COSMOS ${ }^{30}$ results (see table 1).

If we assume that all three XMM based surveys provide correct results, and that their differences are due to the cosmic variance, we can volume weight them to obtain an average estimate of the clustering length of the XMM point sources. The relative volume weights for the XMMCOSMOS, XMM-ELAIS and XMM/2dF surveys are 1:0.9687:0.8527 and 1:0.9947:0.701 for the soft and hard bands, respectively (we have not corrected for the slight differences of the hard-bands used in the three surveys). Doing so we obtain: $\left\langle r_{\circ}\right\rangle \simeq 13.2$ and $16.0 h^{-1} \mathrm{Mpc}$, for the soft and hard bands, respectively. These large correlation lengths are comparable to those of Extremely Red Objects, ${ }^{20}$ of luminous radio sources $\left(; ;^{37} ; 238\right)$ and of bright distant red galaxies. ${ }^{15}$ They are however, significantly larger than those derived from optical AGN surveys: $r_{\circ} \simeq 5.4-8.6 h^{-1} \mathrm{Mpc}$ (eg. $\left.{ }^{12} ;^{27} ; ;^{13} ;{ }^{22}{ }^{3544}\right)$. 


\section{Cosmological Constraints}

It is well known ${ }^{24}$ that according to linear biasing the correlation function of the mass-tracer $\left(\xi_{\mathrm{obj}}\right)$ and dark-matter one $\left(\xi_{\mathrm{DM}}\right)$, are related by:

$$
\xi_{\mathrm{obj}}(r, z)=b^{2}(z) \xi_{\mathrm{DM}}(r, z),
$$

where $b(z)$ is the bias evolution function. In this study we use the bias model of Basilakos \& Plionis, ${ }^{34}$ We quantify the underlying matter distribution clustering by presenting the spatial correlation function of the mass $\xi_{\mathrm{DM}}(r, z)$ as the Fourier transform of the spatial power spectrum $P(k)$ :

$$
\xi_{\mathrm{DM}}(r, z)=\frac{(1+z)^{-(3+\epsilon)}}{2 \pi^{2}} \int_{0}^{\infty} k^{2} P(k) \frac{\sin (k r)}{k r} \mathrm{~d} k,
$$

where $k$ is the comoving wavenumber and $\epsilon=-1.2$, according to the constant in comoving coordinates clustering evolution model. As for the power spectrum, we consider that of CDM models, where $P(k)=P_{0} k^{n} T^{2}(k)$ with scale-invariant $(n=1)$ primeval inflationary fluctuations. In particular, we use the transfer function parameterization as in, ${ }^{2}$ with the corrections given approximately by Sugiyama. ${ }^{41}$ Note that we also use the non-linear corrections introduced by Peacock \& Dodds. ${ }^{33}$ We have chosen to use either the standard normalization given by: $\sigma_{8} \simeq 0.5 \Omega_{\mathrm{m}}^{-\gamma}$ with $\gamma \simeq 0.21-0.22 \mathrm{w}+0.33 \Omega_{\mathrm{m}},{ }^{45}$ or to leave $\sigma_{8}$ a free parameter.

Firstly, using equations (1), (4) and (5) and evaluating at $z=\bar{z}$ we derive the bias factor of our sources at the corresponding redshift. We find $b(\bar{z}) \simeq 5.1 \pm 1.1$ and $\simeq 5.3 \pm 0.9$ for the hard and soft bands respectively, which are significantly smaller than the values attributed to our work in Miyaji et al. ${ }^{30}$

Next, we utilize a $\chi^{2}$ likelihood procedure to compare the measured XMM soft source angular correlation function with the prediction of different spatially flat cosmological models $\left(\right.$ see,$\left.{ }^{56}\right)$. In particular, we define the likelihood estimator as: $\mathcal{L}^{\mathrm{AGN}}(\mathbf{c}) \propto \exp \left[-\chi_{\mathrm{AGN}}^{2}(\mathbf{c}) / 2\right]$ with: $\chi_{\mathrm{AGN}}^{2}(\mathbf{c})=$ $\sum_{i=1}^{n}\left[w_{\text {th }}\left(\theta_{i}, \mathbf{c}\right)-w_{\text {obs }}\left(\theta_{i}\right) / \sigma_{i}\right]^{2}$, where $\mathbf{c}$ is a vector containing the cosmological parameters that we want to fit and $\sigma_{i}$ the observed angular correlation function uncertainty. We assume a flat $\left(\Omega_{\text {tot }}=1\right)$ cosmology with primordial adiabatic fluctuations and baryonic density of $\Omega_{\mathrm{b}} h^{2} \simeq 0.022$ (eg. $\left.;^{2540}\right)$. In this case the corresponding vector is $\mathbf{c} \equiv\left(\Omega_{\mathrm{m}}, \mathrm{w}, \sigma_{8}, h, b_{\circ}\right)$ and we densely sample the various parameters. Note that in order to investigate possible equations of state, we allow the parameter $w$ to take values below -1 , corresponding to the so called phantom cosmologies $\left(\right.$ eg. $\left.{ }^{10}\right)$.

The resulting best fit parameters for $\epsilon=-1.2$ and the Miyaji et $\mathrm{al}^{29}$ luminosity function are presented in Table 2 . In the first two rows we present 
Table 2. Cosmological parameters from the likelihood analysis. Errors of the fitted parameters represent $1 \sigma$ uncertainties. Note that the fitted parameters correspond to results marginalized over the parameters that do not have errorbars, for which we use the values indicated.

\begin{tabular}{cccccc}
\hline Data & $\Omega_{\mathrm{m}}$ & $\sigma_{8}$ & $w$ & $h$ & $b_{\circ}$ \\
\hline XMM & $0.31_{-0.08}^{+0.16}$ & 0.93 & $w=-1$ & $0.72_{-0.18}^{+0.02}$ & $2.30_{-0.20}^{+0.70}$ \\
XMM/SNIa & $0.28 \pm 0.02$ & 0.95 & $-1.05_{-0.20}^{+0.10}$ & 0.72 & 2.30 \\
XMM & $0.28 \pm 0.03$ & $0.75 \pm 0.03$ & $w=-1$ & 0.72 & $2.0_{-0.25}^{+0.20}$ \\
XMM/SNIa & $0.26 \pm 0.04$ & 0.75 & $-0.9_{-0.05}^{+0.10}$ & 0.72 & 2.0 \\
\hline
\end{tabular}

results based on the traditional ${ }^{45} \sigma_{8}$ normalization. Note that our estimate of the Hubble parameter $h$ is in very good agreement with those derived by the HST key project. ${ }^{16}$ In the last two rows of Table 2 we leave $\sigma_{8}$ free but fix the Hubble constant to $h=0.72$. In this case our fit provides a $\sigma_{8}$ value which is in excellent agreement with the recent 3-years WMAP results ${ }^{40}$ Therefore, allowing values $w<-1$ we can derive a $\left(\Omega_{\mathrm{m}}, \sigma_{8}\right)$ relation, a good fit of which is provided by :

$$
\sigma_{8}=0.34( \pm 0.01) \Omega_{\mathrm{m}}^{-\gamma\left(\Omega_{\mathrm{m}}, w\right)}
$$

with $\gamma\left(\Omega_{\mathrm{m}}, w\right)=0.22( \pm 0.04)-0.40( \pm 0.05) w-0.052( \pm 0.040) \Omega_{\mathrm{m}}$. Note that $w$ is degenerate, within the $1 \sigma$ uncertainty, with respect to $\Omega_{\mathrm{m}}$. Therefore, in order to put further constraints on $w$ we additionally use a sample of 172 supernovae SNIa of Tonry et al. ${ }^{42}$

The joined likelihood analysis is performed by marginalizing the X-ray clustering results over $\sigma_{8}, h$ and $b_{0}$. The vector c now becomes: $\mathbf{c} \equiv\left(\Omega_{\mathrm{m}}, w\right)$. The SNIa likelihood function can be written as: $\mathcal{L}^{\mathrm{SNIa}}(\mathbf{c}) \propto \exp \left[-\chi_{\mathrm{SNIa}}^{2}(\mathbf{c}) / 2\right]$, with: $\chi_{\mathrm{SNIa}}^{2}(\mathbf{c})=$ $\sum_{i=1}^{172}\left[\log D_{\mathrm{L}}^{\mathrm{th}}\left(z_{i}, \mathbf{c}\right)-\log D_{\mathrm{L}}^{\mathrm{obs}}\left(z_{i}\right) / \sigma_{i}\right]^{2}$, where $D_{\mathrm{L}}(z)$ is the dimensionless luminosity distance, $D_{\mathrm{L}}(z)=H_{\circ}(1+z) x(z)$ and $z_{i}$ is the observed redshift. The joint likelihood function peaks at: $\Omega_{\mathrm{m}}=0.26 \pm 0.04$ with $w=-0.90_{-0.05}^{+0.1}$. Using eq.(6) we find that the normalization of the power spectrum that corresponds to these cosmological parameters is $\sigma_{8} \simeq 0.73$, in excellent agreement with the recent 3 -year WMAP results. ${ }^{40}$

\section{Acknowledgments}

Many thanks are due to I. Georgantopoulos and A. Georgakakis.

\section{References}

1. Akylas, A., Georgantopoulos, I., Plionis, M., 2000, MNRAS, 318, 1036 
2. Bardeen, J.M., Bond, J.R., Kaiser, N. \& Szalay, A.S., 1986, ApJ, 304, 15

3. Basilakos, S. \& Plionis, M., 2001, ApJ, 550, 522

4. Basilakos, S. \& Plionis, M., 2003, ApJ, 593, L61

5. Basilakos, S. \& Plionis, M., 2005, MNRAS, 360, L35

6. Basilakos, S. \& Plionis, M., 2006, ApJ, 650, L1

7. Basilakos, S., et al., 2004, ApJL, 607, L79

8. Basilakos, S., et al., 2005, MNRAS, 356, 183

9. Boyle, B. J., \& Mo, H. J., 1993, MNRAS, 260, 925

10. Caldell, R. R., 2002, Physics Letters B, 545, 23

11. Carrera, F.J., et al., 1998, MNRAS, 299, 229

12. Croom, S. M., \& Shanks, T., 1996, MNRAS, 281, 893

13. Croom, S.M., et al., 2002, MNRAS, 335, 459

14. de Zotti, G., et al., 1990, ApJ, 351, 22

15. Foucaud, S., et al., 2006, MNRAS, submitted, astro-ph/0606386

16. Freedman, W., L., et al., 2001, ApJ, 553, 47

17. Gandhi, P., et al., 2006, A\&A, 457, 393

18. Georgakakis, A., et al., 2004, MNRAS, 349, 135

19. Georgakakis, A., et al., 2003, MNRAS, 344, 161

20. Georgakakis, A., et al., 2005, ApJ, 620, 584

21. Gilli, R., et al. 2005, A\&A, 430, 811

22. Grazian, A., et al., 2004, AJ, 127, 592

23. Hasinger, G., Miyaji, T., Schmidt, M., 2005, A\&A, 441, 417

24. Kaiser N., 1984, ApJ, 284, L9

25. Kirkman, D., et al., 2003, ApJS, 149, 1

26. Kundić, T., 1997, ApJ, 482, 631

27. La Franca F., Andreani, P., Cristiani, S., 1998, ApJ, 497, 529

28. La Franca, F. et al., 2005, ApJ, 635, 864

29. Miyaji, T., Hasinger, G., Schmidt, M., 2000, A\&A, 353, 25

30. Miyaji, T., et al., 2006, astro-ph/0612369

31. Mullis C.R., et al., 2004, ApJ, 617, 192

32. Overzier, R.A., et al., 2003, A\&A, 405, 53

33. Peacock, A. J., \&, Dodds, S. J., 1994, MNRAS, 267, 1020

34. Peebles P.J.E., 1993. Principles of Physical Cosmology, Princeton Univ.

35. Porciani, C., Magliocchetti, M. \& Norberg, P., 2004, MNRAS,

36. Puccetti, S., et al., 2006, AA submitted, astro-ph/0607107

37. Roche, N. D., Dunlop, J., Almaini, O., 2003, MNRAS, 346, 803

38. Röttgering, H., et al., 2003, New Astr. Rev., 47, 309

39. Schmidt, M., et al., 1998, A\&A, 329, 495

40. Spergel D. N., et al., ApJ, 2006, submitted, astro-ph/0603449

41. Sugiyama, N., 1995, ApJS, 100, 281

42. Tonry, et al. , 2003, ApJ, 594, 1

43. Vikhlinin, A. \& Forman, W., 1995, ApJ, 455, 109

44. Wake, D.A. et al., 2004, ApJ, 610, L85

45. Wang, L. \& Steinhardt, P.J., 1998, ApJ, 508, 483

46. Yang, Y., et al. 2003, ApJ, 585, L85

47. Yang, Y., Mushotzky, R.F., Barger, A.J., Cowie, L.L., 2006, ApJ, 645, 68 